\title{
Diagnostic Value of the Maximum Urethral Closing Pressure in Women With Overactive Bladder Symptoms and Functional Bladder Outlet Obstruction
}

\author{
$\mathrm{Su}$ Jin $\mathrm{Kim}^{1}$, Hee Jung $\mathrm{Choo}^{2}$, Hana Yoon ${ }^{2,3}$ \\ ${ }^{1}$ Department of Urology, College of Medicine, Hallym Unversity, Kangdong Sacred Heart Hospital, Seoul, Korea \\ ${ }^{2}$ Ewha Womans University Seoul Hospital, Seoul, Korea \\ ${ }^{3}$ Department of Urology, Ewha Womans University Seoul Hospital, Ewha Womans University College of Medicine, Seoul, Korea
}

Purpose: Overactive bladder (OAB) is observed in women with functional bladder outlet obstruction (FBOO), as are lower urinary tract symptoms (LUTS). Therefore, we evaluated the urodynamic characteristics of women with storage LUTS and FBOO diagnosed by urodynamic studies (UDS).

Methods: Women $(n=108)$ with storage LUTS and FBOO diagnosed by UDS were included in this study. The urodynamic characteristics of women with $\mathrm{FBOO}$ were compared with those of women with $\mathrm{OAB}(\mathrm{n}=34)$ and stress urinary incontinence (SUI, $\mathrm{n}=78)$. FBOO was defined as a maximum flow rate $(\mathrm{Qmax})<15 \mathrm{~mL} / \mathrm{sec}$, combined with detrusor pressure at a Qmax (PdetQmax) $>20 \mathrm{~cm} \mathrm{H}_{2} \mathrm{O}$ in a pressure-flow study. The UDS included uroflowmetry, postvoid residual volume determination, a cystometrogram, and a pressure-flow study.

Results: Women with FBOO who had storage symptoms showed a higher PdetQmax and maximum urethral closing pressure (MUCP) than women with OAB and SUI. Interstitial cystitis/bladder pain syndrome (IC/BPS) was observed in 17.6\% of women with FBOO. MUCP was significantly higher in women with BOO than in women with BOO and IC/BPS (115.6 \pm 27.4 and $96.6 \pm 14.1 \mathrm{~cm} \mathrm{H}_{2} \mathrm{O}, \mathrm{P}=0.00$ ). The receiver operating characteristic curve analysis showed that the cutoff values of MUCP in women with FBOO compared to women with $\mathrm{OAB}$ and SUI were $87 \mathrm{~cm} \mathrm{H}_{2} \mathrm{O}$ (sensitivity $=81.5 \%$, specificity $=73.5 \%$ ) and $36 \mathrm{~cm} \mathrm{H}_{2} \mathrm{O}$ (sensitivity $=92.6 \%$, specificity $=84.4 \%$ ), respectively. The cutoff value of MUCP in women with FBOO compared to women with FBOO and IC/BPS was $92 \mathrm{~cm} \mathrm{H}_{2} \mathrm{O}$ (sensitivity $=85.4 \%$, specificity $=78.9 \%$ ).

Conclusions: MUCP could have predictive value for identifying FBOO in women with complex LUTS.

Keywords: Women; Lower urinary tract symptoms; Urinary bladder, Overactive; Urinary Bladder Neck Obstruction

- Research Ethics: This study was approved by Institutional Review Board of the Ehwa Womans University Hospital (EUMC2020-01-013).

- Conflict of Interest: No potential conflict of interest relevant to this article was reported.

\section{INTRODUCTION}

Lower urinary tract symptoms (LUTS) represent a common condition associated with diminished quality of life, and more than half of men and women experience some kind of LUTS in their lifetime. In general, the typical symptoms of overactive bladder $(\mathrm{OAB})$ and urinary incontinence (UI) are storage symptoms, and $\mathrm{OAB}$ and $\mathrm{UI}$ are considered to be more prevalent in women than in men. However, women also experience voiding symptoms such as a weak urinary stream, intermitten-

Corresponding author: Hana Yoon (D) https://orcid.org/0000-0002-8553-0152 Department of Urology, Ewha Womans University Seoul Hospital, Ewha Womans University College of Medicine, 260 Gonghang-daero, Gangseo-gu, Seoul 07804, Korea

Email: wowhana@ewha.ac.kr

Submitted: December 30, 2020 / Accepted after revision: May 13, 2021 
cy, and terminal dribbling [1,2]. Therefore, women complaining of voiding symptoms can be diagnosed with female voiding dysfunction (FVD), such as bladder outlet obstruction (BOO) and detrusor underactivity $[3,4]$. Moreover, previous studies have reported that some women experience both storage and voiding symptoms at the same time. Irwin et al. [1] reported that $8 \%$ of women showed both storage and voiding symptoms. Other researchers also showed that $14.5 \%$ of women experienced storage and voiding symptoms and, interestingly, women with voiding symptoms were likely to experience storage and postmicturition symptoms. The reason why women present with complex symptoms such as storage and voiding LUTS is that various conditions such as OAB, UI, and FVD often coexist and symptoms of $\mathrm{OAB}$ can exist secondary to $\mathrm{BOO}$ [5]. Thus, there is a need for research on FVD associated with $\mathrm{BOO}$ in women with storage symptoms.

Clinically, some women with storage LUTS also have BOO; therefore, $\mathrm{OAB}$ treatment fails in these women. According to a study where urodynamic studies (UDS) were performed in women with refractory $\mathrm{OAB}, \mathrm{BOO}$ was observed in $27 \%$ of women and detrusor overactivity (DO) was observed in $38.7 \%$. $\mathrm{BOO}$ was associated with the severity of frequent micturition, nocturia, and a weak urinary stream. Multivariate analysis showed that the severity of frequent micturition was a predictor of BOO [6]. These findings indicate that not all women complaining of symptoms of $\mathrm{OAB}$ are real $\mathrm{OAB}$ patients and that storage symptoms such as frequency and nocturia can be common LUTS in women with BOO. As a result, women with $\mathrm{BOO}$ can be misdiagnosed with $\mathrm{OAB}$, and it is necessary to perform UDS to identify BOO in women.

For women with OAB, UDS is not recommended when considering behavioral and medical treatment [7]. However, a longitudinal prospective follow-up study by Verghese et al. [8] reported improved therapeutic outcomes in women with $\mathrm{OAB}$ symptoms or urgency-predominant mixed UI after they received urodynamic-based treatment. Women with urgencypredominant mixed UI showed better outcomes than women with $\mathrm{OAB}$ symptoms and $\mathrm{DO}$. These results demonstrated that UDS-based treatment decisions help to reduce symptoms and that UDS plays an important role in women with complex symptoms.

There are few studies about the urodynamic characteristics of female BOO patients who are initially diagnosed with $\mathrm{OAB}$ based on their symptoms; therefore, we analyzed urodynamic parameters in women with functional $\mathrm{BOO}$ and investigated differences compared with women with $\mathrm{OAB}$ and with stress urinary incontinence (SUI).

\section{MATERIALS AND METHODS}

This retrospective study (from January 2015 to December 2017) included 108 women (over 20 years old and without any underlying conditions that would influence LUTS) with storage LUTS and functional BOO diagnosed by UDS. All women were initially diagnosed with $\mathrm{OAB}$; however, $\mathrm{OAB}$ treatment failed. Therefore, they received UDS for a functional evaluation. Seventy-eight women with SUI included in this study were diagnosed by a clinical history, physical examination, and UDS. Thirty-four women with OAB who underwent UDS were included. The diagnostic criteria of female $\mathrm{BOO}$ were as follows: maximum flow rate (Qmax) $<15 \mathrm{~mL} / \mathrm{s}$, combined with detrusor pressure at Qmax (PdetQmax) $>20 \mathrm{~cm} \mathrm{H}_{2} \mathrm{O}$ in a pressureflow study. The exclusion criteria were as follows: neurological deficiency; anatomical cause of obstruction; previous pelvic, spinal, or anti-incontinence surgery; intake of drugs affecting voiding; urinary tract infection; and systemic disease (diabetes, hypothyroidism). All patients underwent assessments, including a detailed urogynecological history, a physical examination, urinalysis, urine culture, and multichannel UDS unless the patient responded to anticholinergic treatment. The urodynamic study included uroflowmetry, postvoid residual volume (PVR) determination, a cystometrogram, and a pressure-flow study. The urodynamic techniques and measurements, terms, and diagnostic criteria conformed to the recommendations of the International Continence Society [9].

Statistical analyses were performed using IBM SPSS Statistics ver. 23.0 (IBM Co., Armonk, NY, USA). Analysis of variance was used to determine if significant differences were present among women with $\mathrm{OAB}, \mathrm{BOO}$, and SUI, and the Tukey test was used to determine which groups had significant differences from each other. Additionally, receiver operating characteristic (ROC) analysis was conducted to analyze the parameters that we measured as diagnostic tools. A 5\% level of significance was used for all statistical tests.

\section{RESULTS}

\section{Urodynamic Findings of Women With OAB Versus BOO Versus SUI}

The Qmax, voided volume, and PVR were significantly differ- 
Table 1. Urodynamic parameters in women with OAB, FBOO, and SUI

\begin{tabular}{|c|c|c|c|c|c|c|}
\hline \multirow[b]{2}{*}{ Parameter } & \multirow{2}{*}{$\begin{array}{c}\text { OAB } \\
(n=34)\end{array}$} & \multirow{2}{*}{$\begin{array}{c}\text { SUI } \\
(n=78)\end{array}$} & \multirow{2}{*}{$\begin{array}{l}\text { FBOO } \\
(n=108)\end{array}$} & \multicolumn{3}{|c|}{$\mathrm{P}$-value } \\
\hline & & & & $\begin{array}{c}\text { OAB vs. FBOO } \\
\text { vs. SUI }\end{array}$ & $\begin{array}{c}\text { FBOO vs. } \\
\text { OAB }\end{array}$ & $\begin{array}{c}\text { FBOO vs. } \\
\text { SUI }\end{array}$ \\
\hline \multicolumn{7}{|l|}{ Uroflowmetry } \\
\hline $\operatorname{Qmax}(\mathrm{mL} / \mathrm{sec})$ & $17.8 \pm 10.0$ & $19.8 \pm 8.5$ & $11.7 \pm 6.3$ & 0.00 & 0.00 & 0.00 \\
\hline Voided volume $(\mathrm{mL})$ & $219.2 \pm 133.5$ & $248.2 \pm 109.8$ & $154.9 \pm 111.1$ & 0.01 & - & 0.00 \\
\hline $\operatorname{PVR}(\mathrm{mL})$ & $13.8 \pm 17.1$ & $14.3 \pm 18.1$ & $47.6 \pm 84.3$ & 0.00 & 0.02 & 0.00 \\
\hline \multicolumn{7}{|l|}{ UDS } \\
\hline $\operatorname{MCC}(\mathrm{mL})$ & $399.3 \pm 25.8$ & $387.2 \pm 51.7$ & $335.3 \pm 89.9$ & 0.00 & 0.00 & 0.00 \\
\hline $\mathrm{Qmax}(\mathrm{mL} / \mathrm{sec})$ & $17.6 \pm 7.6$ & $19.6 \pm 6.5$ & $10.0 \pm 6.1$ & - & 0.00 & 0.00 \\
\hline Pdetopen (mL/sec) & $20.9 \pm 8.9$ & $17.0 \pm 7.8$ & $37.1 \pm 26.6$ & - & 0.00 & 0.00 \\
\hline PdetQmax & $23.6 \pm 8.4$ & $18.8 \pm 7.9$ & $39.5 \pm 24.0$ & 0.00 & 0.00 & 0.00 \\
\hline $\operatorname{MUCP}\left(\mathrm{cm} \mathrm{H}_{2} \mathrm{O}\right)$ & $76.2 \pm 24.0$ & $30.2 \pm 7.3$ & $105.0 \pm 39.2$ & 0.00 & 0.00 & 0.00 \\
\hline BOOI & $-11.6 \pm 17.2$ & $-20.4 \pm 17.0$ & $20.6 \pm 27.9$ & 0.00 & 0.00 & 0.00 \\
\hline BCI & $115.0 \pm 34.4$ & $116.8 \pm 31.2$ & $93.6 \pm 32.8$ & 0.00 & 0.00 & 0.00 \\
\hline
\end{tabular}

Values are presented as mean \pm standard deviation.

OAB, overactive bladder; FBOO, functional bladder outlet obstruction; SUI, stress urinary incontinence; UDS, urodynamic study; Qmax, maximum flow rate by uroflowmetry; PVR, postvoid residual volume; UDS, urodynamic study; MCC, maximum cystometric capacity; Pdetopen, detrusor opening pressure; PdetQmax, detrusor pressure at maximum flow rate; MUCP, maximum urethral closing pressure; BOOI, bladder outlet obstruction index; BCI, bladder contractility index.

ent in women with $\mathrm{OAB}, \mathrm{FBOO}$, and SUI. According to UDS, the maximum cystometric capacity (MCC), PdetQmax, and maximum urethral closure pressure (MUCP) were significantly different among the 3 groups. The bladder contractility index and $\mathrm{BOO}$ index were also significantly different (Table 1). DO was noted in $35.2 \%$ (38 of 108) of women with FBOO, but not in the women with $\mathrm{OAB}$.

\section{Comparison of Urodynamic Findings Between Women With BOO and Women With OAB}

A significantly lower Qmax and a higher PVR according to uroflowmetry were observed in women with $\mathrm{FBOO}$ than in women with OAB. According to UDS, the MCC of women with FBOO was significantly lower than that of women with OAB. Moreover, a significantly lower Qmax was noted in women with $\mathrm{FBOO}$ than in women with OAB. The urethral opening pressure (Pdetopen), PdetQmax, and MUCP were significantly higher in women with FBOO than in women with OAB (Table 1).

\section{Comparison of Urodynamic Findings Between Women With BOO and Women With SUI}

A significantly lower Qmax, a lower voided volume, and a higher PVR according to uroflowmetry were observed in wom- en with FBOO than in women with SUI. According to UDS, the MCC of women with FBOO was significantly lower than that of women with SUI. Moreover, a significantly lower Qmax was noted in women with FBOO than in women with SUI. The Pdetopen, PdetQmax, and MUCP were significantly higher in women with FBOO than in women with SUI (Table 1).

\section{Subanalysis of Urodynamic Findings in Women With FBOO}

Eighty-nine of 108 women (82.4\%) showed only FBOO, and 19 women (17.6\%) showed both FBOO and interstitial cystitis/ bladder pain syndrome (IC/BPS). Thirty-eight of 89 women (42.7\%) with FBOO only showed DO. The voided volume and PVR according to uroflowmetry in women with FBOO only were significantly higher than those in women with $\mathrm{FBOO}$ and IC/BPS (Table 2). Moreover, a significantly higher MUCP was observed in women with FBOO only than in women with both FBOO and IC/BPS. However, the MCC was significantly lower in women with $\mathrm{FBOO}$ and IC/BPS than in women with $\mathrm{FBOO}$ only. DO was only noted in women with FBOO only. The ROC analysis between women with FBOO only and women with FBOO and IC/BPS demonstrated that the area under the curve (AUC) of PdetQmax was 0.638 (95\% confidence interval [CI], $0.503-0.773 ; \mathrm{P}=0.067)$. 
Table 2. Urodynamic parameters in women with FBOO only and both FBOO and IC/BPS

\begin{tabular}{|c|c|c|c|}
\hline Parameter & FBOO only $(\mathrm{n}=89)$ & FBOO and IC/BPS $(\mathrm{n}=19)$ & P-value \\
\hline \multicolumn{4}{|l|}{ Uroflowmetry } \\
\hline $\operatorname{Qmax}(\mathrm{mL} / \mathrm{sec})$ & $11.6 \pm 6.2$ & $10.4 \pm 1.8$ & 0.75 \\
\hline Voided volume (mL) & $172.3 \pm 106.6$ & $133.5 \pm 58.7$ & $0.00^{*}$ \\
\hline $\operatorname{PVR}(\mathrm{mL})$ & $33.7 \pm 62.7$ & $24.4 \pm 20.5$ & $0.01^{\star}$ \\
\hline \multicolumn{4}{|l|}{ UDS } \\
\hline $\operatorname{MCC}(\mathrm{mL})$ & $344.9 \pm 91.0$ & $301.6 \pm 77.8$ & $0.01^{\star}$ \\
\hline $\operatorname{Qmax}(\mathrm{mL} / \mathrm{sec})$ & $9.9 \pm 5.9$ & $7.7 \pm 2.8$ & 0.73 \\
\hline Pdetopen & $38.5 \pm 28.0$ & $24.6 \pm 10.0$ & 0.61 \\
\hline PdetQmax & $41.4 \pm 25.2$ & $25.4 \pm 8.3$ & 0.16 \\
\hline $\operatorname{MUCP}\left(\mathrm{cmH}_{2} \mathrm{O}\right)$ & $115.6 \pm 27.4$ & $96.6 \pm 14.1$ & $0.00^{*}$ \\
\hline BOOI & $22.5 \pm 29.3$ & $11.1 \pm 9.2$ & 0.35 \\
\hline BCI & $95.6 \pm 32.1$ & $71.0 \pm 17.4$ & 0.83 \\
\hline
\end{tabular}

Values are presented as mean \pm standard deviation.

FBOO, functional bladder outlet obstruction; IC/BPS, interstitial cystitis/bladder pain syndrome; Qmax, maximum flow rate by uroflowmetry; PVR, postvoid residual volume; UDS, urodynamic study; MCC, maximum cystometric capacity; Pdetopen, detrusor opening pressure; PdetQmax, detrusor pressure at maximum flow rate; MUCP, maximum urethral closing pressure; BOOI, bladder outlet obstruction index; BCI, bladder contractility index.

${ }^{*} \mathrm{P}<0.05$, women with FBOO only vs. FBOO and BPS.
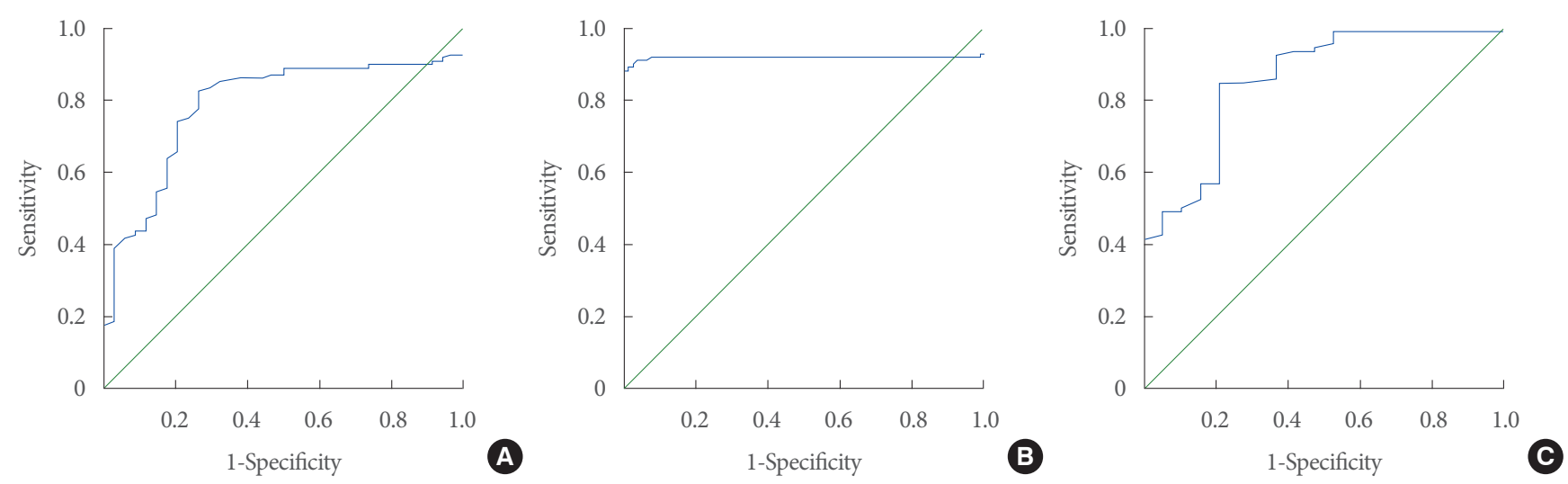

Fig. 1. The receiver operating characteristic curve analysis of MUCP in the women with FBOO versus those with $\mathrm{OAB}(\mathrm{A})$, women with FBOO versus those with SUI (B), and women with FBOO only versus those with both FBOO and IC/BPS (C). The area under the curve of MUCP in women with FBOO versus those with $\mathrm{OAB}$ was 0.786 . The area under the curve of MUCP in women with FBOO versus those with SUI was 0.924 . The area under curve of MUCP in women with FBOO only versus those with both FBOO and IC/BPS was 0.864. Blue line; MUCP, green line; reference line. MUCP, maximum urethral closure pressure; FBOO, functional bladder outlet obstruction; $\mathrm{OAB}$, overactive bladder; IC/BPS, interstitial cystitis/bladder pain syndrome.

\section{Potential Value of MUCP for Predicting FBOO}

The ROC analysis between women with FBOO and women with OAB demonstrated that the AUC of MUCP was 0.786 (95\% CI, 0.698-0.873; $\mathrm{P}=0.000$ ) (Fig. 1A). The AUC of MUCP in women with FBOO compared with women who had SUI was 0.924 (95\% CI, 0.871-0.977; $\mathrm{P}=0.000$ ) (Fig. 1B). The AUC of MUCP in women with FBOO only compared with women who had both FBOO and IC/BPS was 0.864 (95\% CI, 0.7700.958; $\mathrm{P}=0.000$ ) (Fig. 1C). Considering specificity, the cutoff values of FBOO relative to $\mathrm{OAB}$ and SUI were $87 \mathrm{~cm} \mathrm{H}_{2} \mathrm{O}$ and $36 \mathrm{~cm} \mathrm{H}_{2} \mathrm{O}$, respectively. The cutoff value of $\mathrm{FBOO}$ only relative to both $\mathrm{BOO}$ and BPS was $92 \mathrm{~cm} \mathrm{H}_{2} \mathrm{O}$ (Table 3). 
Table 3. Recommendation of the cutoff values of MUCP by ROC analysis

\begin{tabular}{lccc}
\hline MUCP $\left(\mathrm{cm} \mathrm{H}_{2} \mathrm{O}\right)$ & Sensitivity & Specificity & AUC \\
\hline FBOO vs. OAB & & & 0.786 \\
80.5 & 86.1 & 55.9 & \\
85.5 & 82.4 & 73.5 & \\
$87.0^{*}$ & 81.5 & 73.5 & \\
90.5 & 75.0 & 76.5 & \\
FBOO vs. SUI & & & 0.924 \\
30.5 & 92.6 & 44.2 & \\
34.5 & 92.6 & 79.2 & \\
$36.0^{*}$ & 92.6 & 84.4 & \\
38.5 & 92.6 & 90.9 & \\
FBOO only vs. FBOO & & & 0.864 \\
and IC/BPS & & & \\
89.5 & 86.5 & 63.2 & \\
90.5 & 85.4 & 73.7 & \\
$92.0^{*}$ & 85.4 & 78.9 & \\
100.5 & 68.5 & 78.9 & \\
\hline
\end{tabular}

MUCP, maximum closure pressure; ROC, receiver operating characteristics; AUC, area under the ROC curve; FBOO, functional bladder outlet obstruction; $\mathrm{OAB}$, overactive bladder; SUI, stress urinary incontinence; IC/BPS, interstitial cystitis/bladder pain syndrome.

${ }^{\star}$ Recommended cutoff value.

\section{DISCUSSION}

In this study, women with $\mathrm{OAB}$ symptoms and $\mathrm{FBOO}$ who were diagnosed by UDS showed a low Qmax, a lower voiding volume, and a higher PVR than women with SUI or women with OAB. Women with storage symptoms and FBOO showed typical urodynamic findings of BOO, such as an increased PdetQmax and MUCP. The lower MCC and DO observed in women with $\mathrm{FBOO}$ were secondary changes in bladder function induced by BOO. Therefore, these findings induce storage symptoms. Interestingly, $17.6 \%$ of women with FBOO experienced IC/BPS at the same time. Women with FBOO exhibited a higher MUCP than women with FBOO and IC/BPS. In addition, DO was only noted in women with FBOO only. However, women with both FBOO and IC/BPS had a lower MCC than women with FBOO. However, the PdetQmax exhibited low predictive value for differentiating women with both IC/BPS and FBOO from women with FBOO only. Based on these results, for the first time, we suggest MUCP as a potential biomarker for establishing the underlying cause in women who do not respond to symptom-based treatment.

The prevalence of $\mathrm{BOO}$ in women has been reported from $2.7 \%$ to $23 \%$ and is caused by pelvic organ prolapse, urethral stricture, anti-incontinence surgery, FBOO, and dysfunctional voiding [10-14]. Studies on female BOO are not sufficient compared with studies on men with $\mathrm{BOO}$ associated with benign prostatic hyperplasia. However, some studies have investigated the diagnosis and treatment of female BOO. Choi et al. [14] found that $12.8 \%$ of women who visited urologic clinics due to LUTS were diagnosed with female BOO. Their most common LUTS was incomplete bladder emptying, and the second most common symptom was frequency. Moreover, most women with BOO showed both storage and voiding symptoms rather than complaining of isolated voiding or storage symptoms $[10,11]$. Similar to these results, the total International Prostate Symptoms Score, the voiding subscore, and the storage subscore were not significantly different between women with $\mathrm{BOO}$ and $\mathrm{OAB}$ [15]. Symptoms of OAB are sometimes not reliable for diagnosis in women with LUTS [16-18]. Therefore, a detailed evaluation of LUTS and UDS is important to avoid overlooking BOO in women who report OAB symptoms.

Compared with those of $\mathrm{OAB}$ and SUI patients, the characteristic findings of UDS in women with FBOO in the present study were a lower MCC, a lower DO, and a higher MUCP. The lower MCC and DO observed in women with FBOO are factors that induce $\mathrm{OAB}$ symptoms. Several studies have demonstrated that a decreased MCC contributes to inducing storage symptoms. FitzGerald et al. [19] and Sekido et al. [20] observed that frequency and UI were associated with a lower MCC in women with OAB. In addition, Cho et al. [14] found a lower MCC in women with BOO, similar to the results of the present study. Although few studies have investigated DO in women with BOO, 2 previous studies showed that DO was observed in $12.6 \%$ and $49.9 \%$ of women with BOO, respectively $[6,16]$. In the present study, DO was noted in $35.2 \%$ of women with FBOO. DO is associated with urgency and urge UI as well as symptom severity $[6,15]$. Therefore, women with DO induced by $\mathrm{BOO}$ are likely to experience $\mathrm{OAB}$ symptoms. In general, the diagnostic role of MUCP is considered low, and no studies have assessed the value of MUCP for predicting female BOO. We noted that MUCP in women with SUI $\left(30.2 \pm 7.3 \mathrm{~cm} \mathrm{H}_{2} \mathrm{O}\right)$ was similar to that in previous reports by Weber (women with SUI: $39.4 \pm 24 \mathrm{~cm} \mathrm{H}_{2} \mathrm{O}$ ) [21]. Moreover, MUCP in women with $\mathrm{FBOO}$ was significantly higher than that in women with $\mathrm{OAB}$ and SUI; therefore, MUCP might have diagnostic value. Al- 
though PdetQmax has diagnostic value for discriminating BOO in women, some patients cannot complete the pressureflow study due to emotional distress. Thus, MUCP may help to diagnose $\mathrm{BOO}$ in women who cannot urinate during a pressure-flow study [22].

Interestingly, 19\% of women with FBOO had IC/BPS, and this is the first report of IC/BPS found in women with FBOO. In contrast, some investigators have analyzed the urodynamic characteristics of women with BOO and IC/BPS. Cameron and Gajewski [23] observed that $48.1 \%$ of women with IC/BPS had $\mathrm{BOO}$ according to a pressure-flow study, and others reported that $60.1 \%$ of women with IC/BPS showed symptoms of bladder outlet dysfunction, such as dysfunctional voiding, poor relaxation of the external urethral sphincter, and bladder neck dysfunction, according to a video urodynamic study [24]. In addition, their IC/BPS patients with BOO showed a lower bladder capacity, a smaller voided volume, a larger PVR, a higher PdetQmax, and a lower Qmax than IC/BPS women without BOO. Similar to these previous findings, women with FBOO and IC/BPS in the current study showed a lower voided volume and bladder capacity than women with FBOO only. However, a lower MUCP was observed in women with FBOO and IC/BPS than in women with FBOO only. Moreover, the Qmax and PdetQmax were not significantly different. From these results, we infer that the decreased bladder capacity in women with FBOO and IC/BPS was associated with pain in comparison with women with FBOO only. The lower MUCP in the women with FBOO and IC/BPS might have also been influenced by IC/BPS. There have not been enough studies defining the underlying mechanism of the coexistence of BOO and IC/BPS; however, some researchers have proposed possible hypotheses such as pelvic floor hypertonicity. Pelvic floor spasms and impairments in pelvic muscle relaxation have been observed in both BOO and IC/BPS [25,26].

Women with FBOO had significantly different MUCP values from those in women with OAB and SUI. Moreover, a significant difference in MUCP was noted between women with FBOO only and women with FBOO and IC/BPS. Therefore, MUCP might be a potential biomarker for FBOO in women with $\mathrm{OAB}$ symptoms. From these results, we suggest a cutoff value of MUCP for the prediction of FBOO. In OAB women, an MUCP $\geq 87 \mathrm{~cm} \mathrm{H}_{2} \mathrm{O}$ might be a predictor of $\mathrm{FBOO}$, and in women with SUI, an MUCP $\geq 36 \mathrm{~cm} \mathrm{H}_{2} \mathrm{O}$ might be a predictor of FBOO. Since PdetQmax had poor predictive value for the differentiation of women with FBOO and IC/BPS from women with FBOO without IC/BPS, an MUCP $\geq 92 \mathrm{~cm} \mathrm{H}_{2} \mathrm{O}$ might be a predictor for $\mathrm{FBOO}$ in women with $\mathrm{FBOO}$ and IC/BPS. Although we found a possible role for MUCP as a prediction tool, there are limitations to the general application of our suggested cutoff value of MUCP because the current definition of FBOO is diverse among clinicians. However, MUCP could help to identify FBOO in women with complex LUTS.

In conclusion, women with storage symptoms and FBOO showed different urodynamic findings compared with women with $\mathrm{OAB}$ and women with SUI. Moreover, some women simultaneously had FBOO and IC/BPS. Therefore, special interest in patients who complain of LUTS is necessary because LUTS is associated with various underlying conditions, and UDS should be performed in patients in whom OAB medication fails. For the first time, we reported that MUCP could have predictive value for identifying FBOO in women with complex LUTS.

\section{AUTHOR CONTRIBUTION STATEMENT}

- Conceptualization: $H Y$

- Data curation: SJK, HJC

- Funding acquisition: $H Y$

- Methodology: HY

- Project administration: $H Y$

- Visualization: SJK

-Writing-original draft: $S J K$

-Writing-review \& editing: SJK, $H Y$

\section{ORCID}

Su Jin Kim

0000-0002-1917-2780

Hee Jung Choo

0000-0002-3397-3613

Hana Yoon

0000-0002-8553-0152

\section{REFERENCES}

1. Irwin DE, Milsom I, Hunskaar S, Reilly K, Kopp Z, Herschorn S, et al. Population-based survey of urinary incontinence, overactive bladder, and other lower urinary tract symptoms in five countries: results of the EPIC study. Eur Urol 2006;50:1306-14.

2. Park J, Kim ES, Lee YJ, Lee HS, Seo JT. Sex differences in lower urinary tract symptoms in older korean adults living in rural areas: prevalence, quality of life, and associated factors. Int Neurourol J 2018;22:212-9. 
3. Robinson D, Staskin D, Laterza RM, Koelbl H. Defining female voiding dysfunction: ICI-RS 2011. Neurourol Urodyn 2012;31:3136.

4. Mytilekas KV, Oeconomou A, Sokolakis I, Kalaitzi M, Mouzakitis G, NakopoulouE, et al. Defining voiding dysfunction in women: bladder outflowobstruction versus detrusor underactivity. Int Neurourol J 2021;25:244-51.

5. Coyne KS, Sexton CC, Thompson CL, Milsom I, Irwin D, Kopp $\mathrm{ZS}$, et al. The prevalence of lower urinary tract symptoms (LUTS) in the USA, the UK and Sweden: results from the Epidemiology of LUTS (EpiLUTS) study. BJU Int 2009;104:352-60.

6. Al-Zahrani AA, Gajewski J. Urodynamic findings in women with refractory overactive bladder symptoms. Int J Urol 2016;23:75-9.

7. Scarpero H. Urodynamics in the evaluation of female LUTS: when are they helpful and how do we use them? Urol Clin North Am 2014;41:429-38.

8. Verghese TS, Middleton LJ, Daniels JP, Deeks JJ, Latthe PM. The impact of urodynamics on treatment and outcomes in women with an overactive bladder: a longitudinal prospective follow-up study. Int Urogynecol J 2018;29:513-9.

9. Abrams P, Cardozo L, Fall M, Griffiths D, Rosier P, Ulmsten U, et al. The standardisation of terminology of lower urinary tract function: report from the Standardisation Sub-committee of the International Continence Society. Am J Obstet Gynecol 2002;187:11626.

10. Blaivas JG, Groutz A. Bladder outlet obstruction nomogram for women with lower urinary tract symptomatology. Neurourol Urodyn 2000;19:553-64.

11. Kim DK, Lee JY, Jung JH, Kim JH, Hah YS, Hong CH, et al. Alpha-1 adrenergic receptor blockers for the treatment of lower urinary tract symptoms in women: a systematic review and metaanalysis. Int Neurourol J 2019;23:56-68.

12. Chassagne S, Bernier PA, Haab F, Roehrborn CG, Reisch JS, Zimmern PE. Proposed cutoff values to define bladder outlet obstruction in women. Urology 1998;51:408-11.

13. Nitti VW, Tu LM, Gitlin J. Diagnosing bladder outlet obstruction in women. J Urol 1999;161:1535-40.

14. Choi YS, Kim JC, Lee KS, Seo JT, Kim HJ, Yoo TK, et al. Analysis of female voiding dysfunction: a prospective, multicenter study. Int Urol Nephrol 2013;45:989-94.
15. Cho KJ, Kim HS, Koh JS, Kim JC. Evaluation of female overactive bladder using urodynamics: relationship with female voiding dysfunction. Int Braz J Urol 2015;41:722-8.

16. Kuo HC. Clinical symptoms are not reliable in the diagnosis of lower urinary tract dysfunction in women. J Formos Med Assoc 2012;111:386-91.

17. Daan NM, Schweitzer KJ, van der Vaart CH. Associations between subjective overactive bladder symptoms and objective parameters on bladder diary and filling cystometry. Int Urogynecol J 2012;23: 1619-24.

18. Hsiao SM, Wu PC, Chang TC, Chen CH, Lin HH. Urodynamic and bladder diary factors predict overactive bladder-wet in women: a comparison with overactive bladder-dry. Int Neurourol J 2019; 23:69-74.

19. FitzGerald MP, Butler N, Shott S, Brubaker L. Bother arising from urinary frequency in women. Neurourol Urodyn 2002;21:36-40.

20. Sekido N, Hinotsu S, Kawai K, Shimazui T, Akaza H. How many uncomplicated male and female overactive bladder patients reveal detrusor overactivity during urodynamic study? Int J Urol 2006;13: 1276-9.

21. Weber AM. Is urethral pressure profilometry a useful diagnostic test for stress urinary incontinence? Obstet Gynecol Surv 2001;56: 720-35.

22. Suskind AM, Clemens JQ, Kaufman SR, Stoffel JT, Oldendorf A, Malaeb BS, et al. Patient perceptions of physical and emotional discomfort related to urodynamic testing: a questionnaire-based study in men and women with and without neurologic conditions. Urology 2015;85:547-51.

23. Cameron AP, Gajewski JB. Bladder outlet obstruction in painful bladder syndrome/interstitial cystitis. Neurourol Urodyn 2009;28: 944-8.

24. Kuo YC, Kuo HC. Videourodynamic characteristics of interstitial cystitis/bladder pain syndrome-The role of bladder outlet dysfunction in the pathophysiology. Neurourol Urodyn 2018;37:1971-7.

25. Deindl FM, Vodusek DB, Bischoff C, Hofmann R, Hartung R. Dysfunctional voiding in women: which muscles are responsible? Br J Urol 1998;82:814-9.

26. Butrick CW, Sanford D, Hou Q, Mahnken JD. Chronic pelvic pain syndromes: clinical, urodynamic, and urothelial observations. Int Urogynecol J Pelvic Floor Dysfunct 2009;20:1047-53. 